\title{
The Nature of the Light Variation of the Unique Binary DK CVn
}

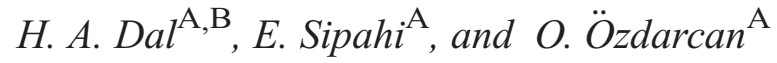 \\ A Department of Astronomy and Space Sciences, University of Ege, Bornova, 35100 İzmir, Turkey \\ ${ }^{\mathrm{B}}$ Corresponding author. Email: ali.dal@ege.edu.tr
}

\begin{abstract}
We present $B V R$ observations of DK CVn from 2007 and 2008 . We analysed the $B V R$ light curves of the system and obtained the system's parameters. Using the ' $q$-search' method, we measured the mass ratio of the system $(q)$ as 0.55 . Taking the temperature of the primary component as $4040 \mathrm{~K}$, the temperature of the secondary was found to be $3123 \mathrm{~K}$. Several flares were detected, and the distributions of flare equivalent duration versus flare total duration were modelled using the One-Phase Exponential Association Function for these flares. The parameters of the model demonstrated that the flares are the same as those detected from UV Ceti stars. We also demonstrate that the variation at out-of-eclipse must be caused by some cool spot(s) on one of the components. The star is found to show two active longitudes in which the spots are mainly formed. Consequently, this study reveals that DK CVn should be a chromospherically active binary star.
\end{abstract}

Keywords: binaries: close — stars: activity — stars: individual: DK CVn — stars: flare — stars: spots

Received 2011 August 2, accepted 2011 November 30, published online 2011 December 22

\section{Introduction}

DK Canum Venaticorum (=GSC 03018 01509) is classified as an eclipsing binary star in the SIMBAD database. The system was first discovered by the Robotic Optical Transient Search Experiment (Akerlof et al. 2000).

Examining the light variation of the system, Diethelm (2001) noted that the reflection effect dominates the light variation. DK CVn was identified as a variable star by Kazarovets et al. (2003) and was listed as an Algol-type variable (EA) in the Catalogue of Eclipsing Variables by Malkov et al. (2006). Terrell et al. (2005) noticed from $U B V R_{\mathrm{C}} I_{\mathrm{C}}$-band observations of the season 2002 that the amplitudes of the humps around 0.25 increase from the $I_{\mathrm{C}}$ band to $U$ band.

A flare was detected by Terrell et al. (2005) in the observations of the 2003 season. Using low-resolution spectroscopy, they determined that the primary component is from $\mathrm{K} 7$ spectral type. Considering the light curves obtained in $I_{\mathrm{C}}$ band, they indicate that the secondary component must be a star from the late-M spectral type.

In this study, $B V R$ photometry of DK CVn was taken in 2007 and 2008. All the details of the observations and reduction procedures are given in Section 2.1. The new light elements are given in Section 2.2. Using v0.31a of PHOEBE software (Prša \& Zwitter 2005), the BVR light curves were analysed, and some physical parameters were obtained. The details of the analysis are described in Sections 2.3 and 2.4. Extracting the obtained synthetic light curve from the observed light curves, it was examined whether there is any out-of-eclipse variation. All the procedures are described in Section 2.5. Several flares were detected. Some parameters were computed for each flare. A One-Phase Exponential Association (hearafter OPEA) model was derived for the flares. The analyses and the OPEA model of the flares are described in Section 2.6. All the results are given and discussed in Section 3.

\section{Observations and Analyses}

\subsection{Observations}

Observations were acquired with a thermoelectrically cooled ALTA U + $422048 \times 2048$-pixel CCD camera attached to a 40-cm Schmidt-Cassegrain MEADE telescope at Ege University Observatory. The BVR-band observations were recorded over two nights in 2007 and five nights in 2008. Some basic parameters of program stars are listed in Table 1 (in this table, the spectral types are taken from Tokunaga 2000).

Although the program and comparison stars are very close on the sky, differential atmospheric-extinction corrections were applied. The atmospheric-extinction coefficients were obtained from observations of the comparison stars on each night. Moreover, the comparison stars were observed with the standard stars in their vicinity and the reduced differential magnitudes, in the sense variable-minus-comparison, were transformed to the standard system using procedures outlined by Hardie (1962). The standard stars are listed in catalogues of Landolt (1983, 1992). Furthermore, the dereddened colours of the system were computed. Heliocentric corrections were also applied to the times of the observations.

The mean averages of the standard deviations are $0.021 \mathrm{mag}, 0.013 \mathrm{mag}$, and $0.019 \mathrm{mag}$ for observations 
Table 1. Basic parameters for the observed stars

\begin{tabular}{|c|c|c|c|c|}
\hline Star & Alpha/Delta (J2000) & $V(\mathrm{mag})$ & $B-V$ (mag) & Spectral type \\
\hline DK CVn & $12^{\mathrm{h}} 33^{\mathrm{m}} 09 \$ 34 /+37^{\circ} 58^{\prime} 20^{\prime \prime} .28$ & 12.967 & 0.890 & $\mathrm{~K} 2$ \\
\hline GSC 30182499 & $12^{\mathrm{h}} 33^{\mathrm{m}} 11^{\mathrm{s}} 14 /+37^{\circ} 45^{\prime} 12^{\prime \prime} .90$ & 12.004 & 0.527 & F8 \\
\hline GSC 30182425 & $12^{\mathrm{h}} 32^{\mathrm{m}} 58.36 /+37^{\circ} 54^{\prime} 20^{\prime \prime} .30$ & 12.702 & 0.628 & G2 \\
\hline
\end{tabular}

(a)

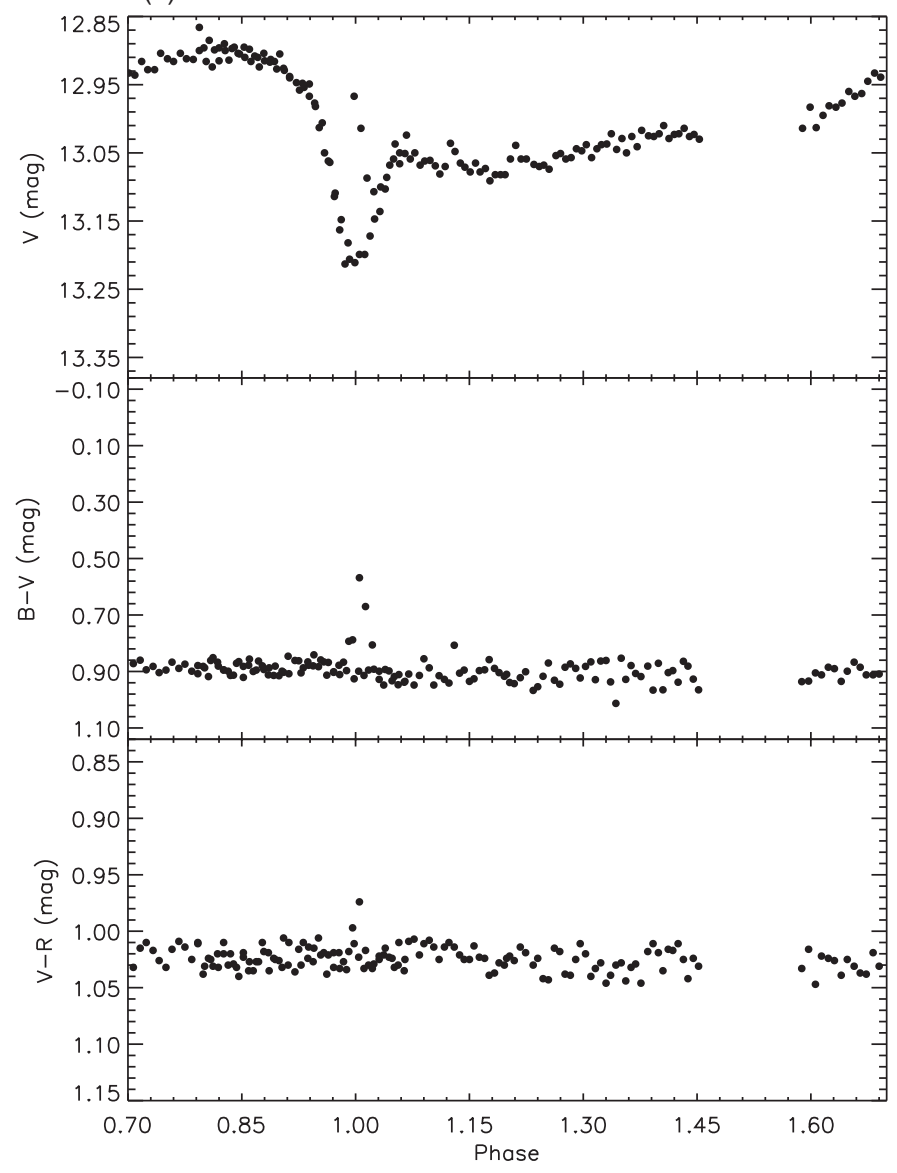

(b)

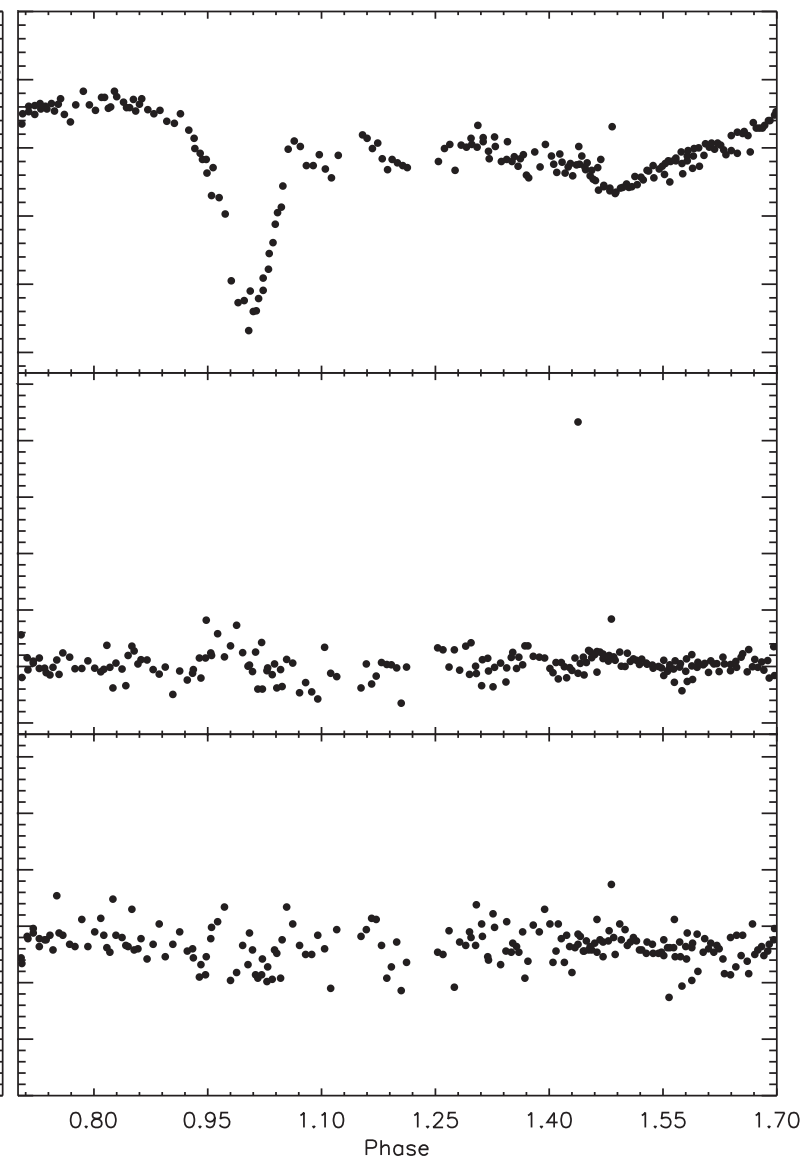

Figure 1 The $V$-band light curve with both $B-V$ and $V-R$ colour curves of DK CVn from (a) the 2007 observations and (b) the 2008 observations.

acquired in the $B, V$ and $R$ bands, respectively. To compute the standard deviations of observations, we used the standard deviations of the reduced differential magnitudes in the sense comparisons minus check stars for each night. There was no variation observed in the standard brightnesses of the comparison stars.

In Figure 1, the light and colour curves of DK CVn are shown. Comparing the 2007 and 2008 light curves, it can be seen that there are some differences between two light curves. The decreases in the levels of both maxima and minima of 2007 and 2008 light curves are seen, and the shapes of the light curves also changed from 2007 to 2008. The parts of the light curves between 0.70 and 1.20 are especially different from each other. On the other hand, there is no variation in the colour curves except the flare moments. Moreover, there are no variations in the mean colour, while there are some variations in the mean brightness. The secondary minimum was not obtained in 2007 due to the absence of the observation in those phases. In Figure 2, there are two $V$-band light curves, whose data were taken from the database of The Northern Sky Variability Survey (hereafter NSVS) (Woźniak et al. 2004). As seen from Figure 2, the shape of DK CVn's light curve is rapidly varying, even in one observing season.

\subsection{Times of Minima and Orbital Period}

We could find 54 times of minima (included both primary and secondary) from the literature. The data cover an interval from 2001 to the current time and listed in Table 2. All the minimum times used in the analysis were obtained in photoelectric observations. Using the linear least-squares method, an update linear ephemeris is given by:

$$
H J D=2453422.9838(2)+0 \stackrel{d}{\mathrm{~d}} 494964(1) E .
$$




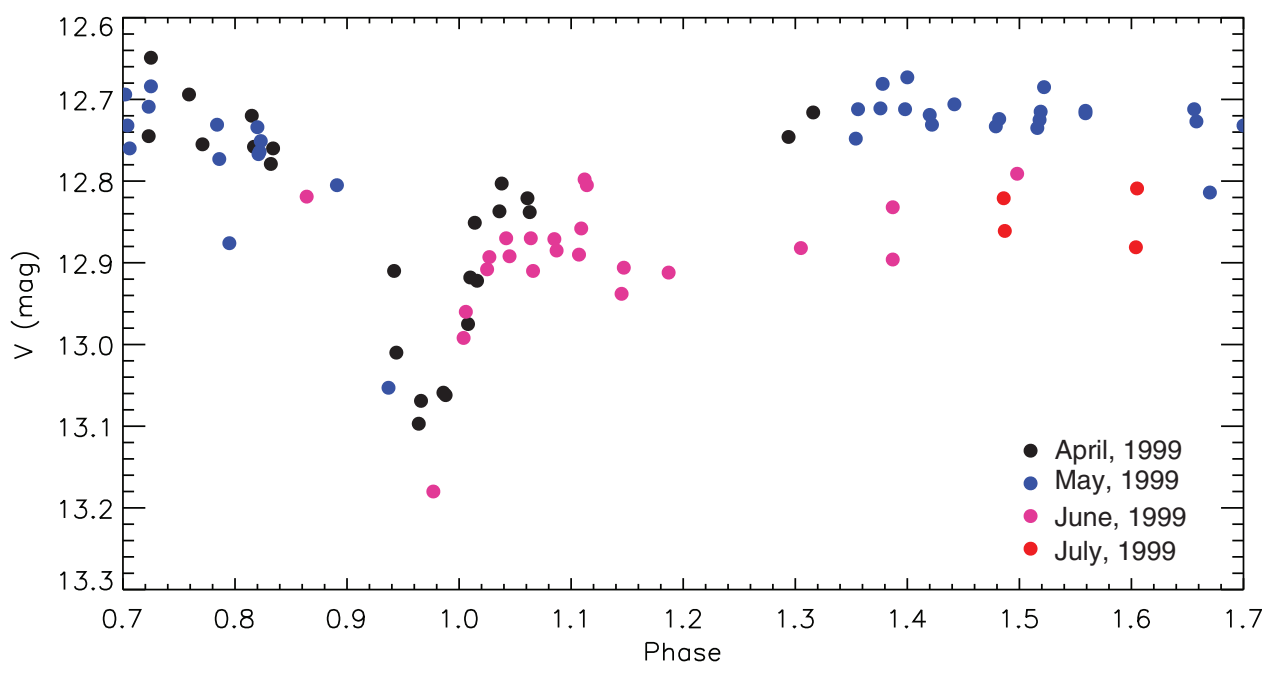

Figure 2 The $V$-band light curves of the observing season 1999. The data were taken from the NSVS database (Woźniak et al. 2004).

The linear correction $(O-C)_{\mathrm{I}}$ is shown in Figure 3. The phases in all the figures are calculated with these new light elements.

\subsection{Light-Curve Analysis}

Although the secondary minimum was not obtained in 2007, the whole light curve was obtained in 2008. Because of this, we analysed the $B V R$ light curves obtained in 2008. The analyses were carried out with using PHOEBE software v0.31a (Prša \& Zwitter 2005). The method used by PHOEBE uses the method used in the 2003 version of the Wilson-Devinney Code (Wilson \& Devinney 1971; Wilson 1990). The BVR light curves were analysed with the 'detached system', 'semidetached system with the primary component filling its Roche-Lobe' and 'semi-detached system with the secondary component filling its Roche-Lobe' modes. An acceptable result was only obtained in 'detached-system' mode, while no acceptable results were obtained in all the others modes.

Terrell et al. (2005), who examined the low resolution spectrum of the system, demonstrated that the primary component is a $\mathrm{K} 7 \mathrm{~V}$ star. We took $J H K$ brightness of the system $(J=10.489 \mathrm{mag}, H=9.839 \mathrm{mag}$ and $K=9.664$ mag) from the NOMAD Catalogue (Zacharias et al. 2004). Using these brightnesses, we derived dereddened colours as a $(J-H)_{\mathrm{o}}=0.561 \mathrm{mag}$ and $(H-K)_{\mathrm{o}}=0.140$ mag for the system. Using the calibrations given by Tokunaga (2000), we derived the temperature of the primary component as $4040 \mathrm{~K}$ depending on these dereddened colours. The derived temperature is in agreement with the spectral type given by Terrell et al. (2005). In the analyses, the temperature of the primary component was fixed to $4040 \mathrm{~K}$, and the temperature of the secondary was taken as a free parameter.

Considering the spectral types, we adopted albedos $\left(A_{1}\right.$ and $\left.A_{2}\right)$ and gravity-darkening coefficients $\left(g_{1}\right.$ and $\left.g_{2}\right)$ for stars with convective envelopes (Lucy 1967; Rucinski 1969). The non-linear limb-darkening coefficients $\left(x_{1}\right.$ and $\left.x_{2}\right)$ of the components were taken from van Hamme (1993). In the analyses, their dimensionless potentials $\left(\Omega_{1}\right.$ and $\left.\Omega_{2}\right)$, the fractional luminosity $\left(L_{1}\right)$ of the primary component and the inclination $(i)$ of the system were taken as the adjustable free parameters.

In order to find the best photometric mass ratio of the components, we used the ' $q$-search' method with a step of 0.05 , due to the absence of any spectroscopic mass ratios. As seen from Figure 4, the minimum sum of weighted squared residuals $\left(\sum \mathrm{res}^{2}\right)$ is found for a mass ratio $q=0.55$. According to this result, we believe that a possible mass ratio of the system is $q=0.55$.

As it is clearly seen from Figure 1, there is the clear asymmetry in the light curves of both 2007 and 2008 . Moreover, the shape of the asymmetric light curve changed from 2007 to 2008 . In order to remove the asymmetry, we assumed that the primary component has two cool spots on its surface. The synthetic light curves obtained from the best light-curve solution are seen in Figure 5, and the result parameters of the analysis are also listed in Table 3. The 3D model of Roche geometry is shown in Figure 6.

\subsection{Estimated Absolute Parameters}

Although there is not any radial-velocity curve for this system, we tried to estimate the absolute parameters of the components. Considering its spectral type, we took the mass of the primary component from Tokunaga (2000), and the mass of the secondary component was calculated from the estimated mass ratio of the system ( 0.55$)$. Using Kepler's third law, we calculated the semi-major axis $(a)$, and then the mean radii of the components were calculated. The mass of the components were found to be $M_{1}=0.44 M_{\odot}$, and it $M_{2}=0.24 M_{\odot}$. Considering the estimated $a$ value, the radii of the two components were computed to be $R_{1}=0.58 R_{\odot}$ and $R_{2}=0.59 R_{\odot}$. Using the estimated radii and the obtained temperatures of the components, the component luminosities were calculated to be $L_{1}=0.08 L_{\odot}$ and $L_{2}=0.03 L_{\odot}$. 
Table 2. Minima times and $O-C$ residuals

\begin{tabular}{|c|c|c|c|c|c|}
\hline HJD (+2 400000$)$ & $E$ & $(O-C)_{\mathrm{I}}$ & $(O-C)_{\mathrm{II}}$ & Filter & Ref. \\
\hline 52001.4487 & -2872.0 & -0.0019 & 0.0010 & CCD & 1 \\
\hline 52361.7820 & -2144.0 & -0.0017 & 0.0006 & $R$ & 2 \\
\hline 52363.7604 & -2140.0 & -0.0031 & -0.0008 & $I$ & 2 \\
\hline 52363.7618 & -2140.0 & -0.0017 & 0.0006 & $R$ & 2 \\
\hline 52363.7623 & -2140.0 & -0.0012 & 0.0011 & $V$ & 2 \\
\hline 52408.8049 & -2049.0 & -0.0003 & 0.0020 & $\mathrm{U}$ & 2 \\
\hline 52693.9027 & -1473.0 & -0.0012 & 0.0006 & CCD & 2 \\
\hline 52712.7104 & -1435.0 & -0.0021 & -0.0003 & $R$ & 2 \\
\hline 52713.7008 & -1433.0 & -0.0016 & 0.0002 & $R$ & 2 \\
\hline 53082.4493 & -688.0 & -0.0005 & 0.0006 & $\mathrm{CCD}$ & 2 \\
\hline 53083.9362 & -685.0 & 0.0015 & 0.0026 & $R$ & 2 \\
\hline 53085.9135 & -681.0 & -0.0011 & 0.0000 & $V$ & 2 \\
\hline 53094.8234 & -663.0 & -0.0005 & 0.0006 & $V$ & 2 \\
\hline 53108.6837 & -635.0 & 0.0008 & 0.0019 & $I$ & 2 \\
\hline 53109.6731 & -633.0 & 0.0003 & 0.0014 & $B$ & 2 \\
\hline 53383.8786 & -79.0 & -0.0037 & -0.0031 & $V$ & 2 \\
\hline 53383.8788 & -79.0 & -0.0035 & -0.0029 & $V$ & 2 \\
\hline 53383.8798 & -79.0 & -0.0025 & -0.0019 & $R$ & 2 \\
\hline 53385.8600 & -75.0 & -0.0022 & -0.0015 & $V$ & 2 \\
\hline 53388.8299 & -69.0 & -0.0020 & -0.0014 & $R$ & 2 \\
\hline 53389.8191 & -67.0 & -0.0028 & -0.0022 & $R$ & 2 \\
\hline 53390.8090 & -65.0 & -0.0028 & -0.0022 & $R$ & 2 \\
\hline 53420.0139 & -6.0 & -0.0007 & -0.0001 & $B$ & 2 \\
\hline 53421.9926 & -2.0 & -0.0019 & -0.0013 & $B$ & 2 \\
\hline 53422.9834 & 0.0 & -0.0010 & -0.0004 & $B$ & 2 \\
\hline 53426.9420 & 8.0 & -0.0021 & -0.0015 & $B$ & 2 \\
\hline 53427.9322 & 10.0 & -0.0018 & -0.0013 & $B$ & 2 \\
\hline 53430.9040 & 16.0 & 0.0002 & 0.0007 & $V$ & 2 \\
\hline 53430.9043 & 16.0 & 0.0005 & 0.0010 & $B$ & 2 \\
\hline 53432.8825 & 20.0 & -0.0012 & -0.0006 & $B$ & 2 \\
\hline 53432.8840 & 20.0 & 0.0003 & 0.0009 & V & 2 \\
\hline 53433.8726 & 22.0 & -0.0010 & -0.0004 & $B$ & 2 \\
\hline 53443.7712 & 42.0 & -0.0016 & -0.0011 & $V$ & 2 \\
\hline 53445.7507 & 46.0 & -0.0020 & -0.0015 & $B$ & 2 \\
\hline 53448.7210 & 52.0 & -0.0015 & -0.0010 & $B$ & 2 \\
\hline 53451.6915 & 58.0 & -0.0007 & -0.0002 & $V$ & 2 \\
\hline 53456.6409 & 68.0 & -0.0010 & -0.0005 & $R$ & 2 \\
\hline 53457.6302 & 70.0 & -0.0016 & -0.0011 & $B$ & 2 \\
\hline 53478.9140 & 113.0 & -0.0012 & -0.0007 & $R$ & 2 \\
\hline 53479.9051 & 115.0 & 0.0000 & 0.0004 & $B$ & 2 \\
\hline 53496.7330 & 149.0 & -0.0009 & -0.0004 & $R$ & 2 \\
\hline 54168.4007 & 1506.0 & 0.0020 & 0.0013 & $B V R$ & 3 \\
\hline 54217.4049 & 1605.0 & 0.0049 & 0.0041 & $V R$ & 3 \\
\hline 54220.3719 & 1611.0 & 0.0021 & 0.0013 & $R$ & 4 \\
\hline 54220.3725 & 1611.0 & 0.0027 & 0.0019 & $V R$ & 3 \\
\hline 54531.4535 & 2239.5 & -0.0006 & -0.0019 & $V$ & 5 \\
\hline 54531.4553 & 2239.5 & 0.0012 & -0.0001 & $I$ & 5 \\
\hline 54531.4557 & 2239.5 & 0.0016 & 0.0003 & $R$ & 5 \\
\hline 54564.3733 & 2306.0 & 0.0042 & 0.0029 & $B V R$ & 3 \\
\hline 54619.3154 & 2417.0 & 0.0054 & 0.0040 & $V R$ & 3 \\
\hline 54929.4066 & 3043.5 & 0.0023 & 0.0003 & $R$ & 4 \\
\hline 54936.3379 & 3057.5 & 0.0041 & 0.0021 & $\mathrm{CCD}$ & 4 \\
\hline 55691.4004 & 4583.0 & 0.0005 & -0.0028 & $V R$ & 6 \\
\hline 55697.3413 & 4595.0 & 0.0018 & -0.0014 & $R I$ & 6 \\
\hline
\end{tabular}

${ }^{1}$ Brát, Zejda \& Svoboda (2007)

${ }^{2}$ Terrell et al. (2005)

${ }^{3}$ Sipahi et al. (2009)

${ }^{4}$ Brát et al. (2009)

${ }^{5}$ Brát et al. (2008)

${ }^{6}$ This study. 


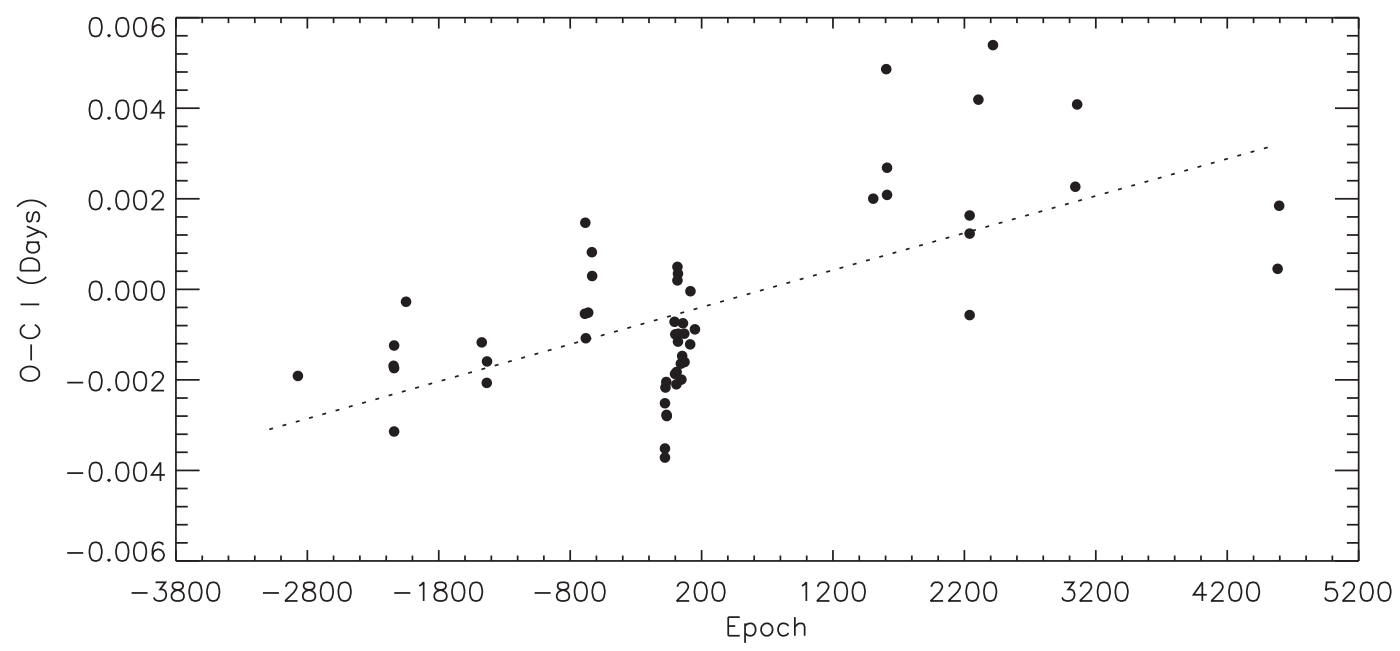

Figure 3 The $O-C$ diagram of DK CVn (the dashed line represents the linear fit).

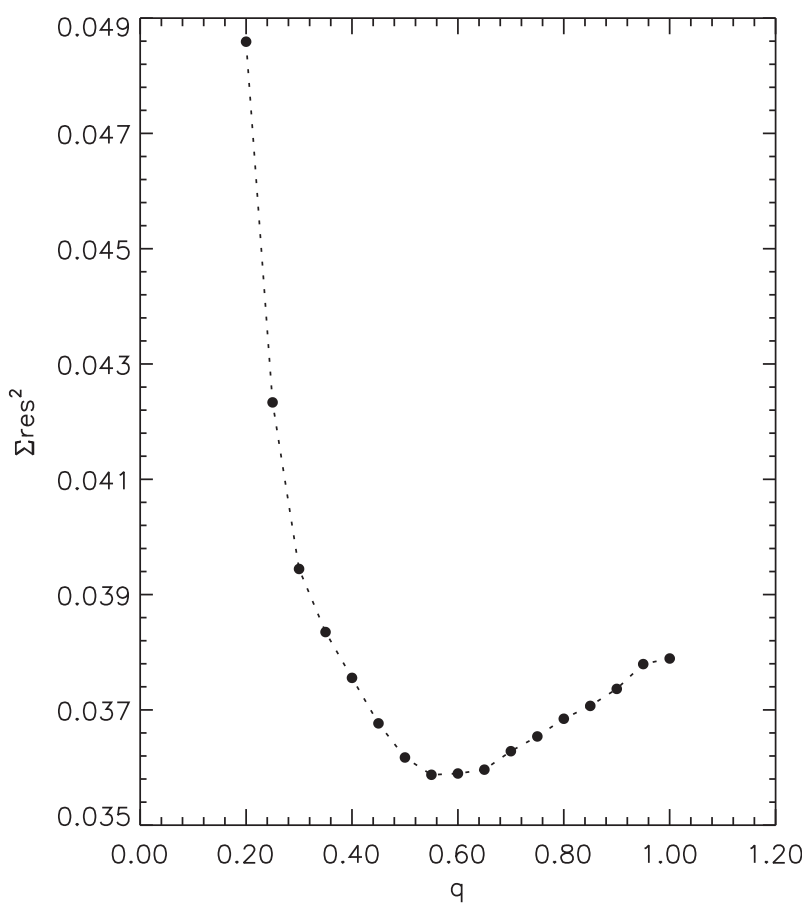

Figure 4 The variation of the sum of weighted, squared residuals versus mass ratio in $q$-search.

The absolute parameters seem to be reasonable for this type of star. However, the calculated radii of both components are larger than the expected values given by theoretical models. In Figure 7, we plot the distribution of radius versus mass for some stars. In the figure, the filled circles represent the known active stars, which were taken from the catalogue of Gershberg et al. (1999). Some of these stars exhibit spot activity, while some of them exhibit flare activity. Some stars exhibit both spot and flare activities. In the figure, the asterisk represents the secondary component of DK CVn, while the open triangle represents the primary component. The line represents the ZAMS theoretical model developed by Siess, Dufour \& Forestini (2000).

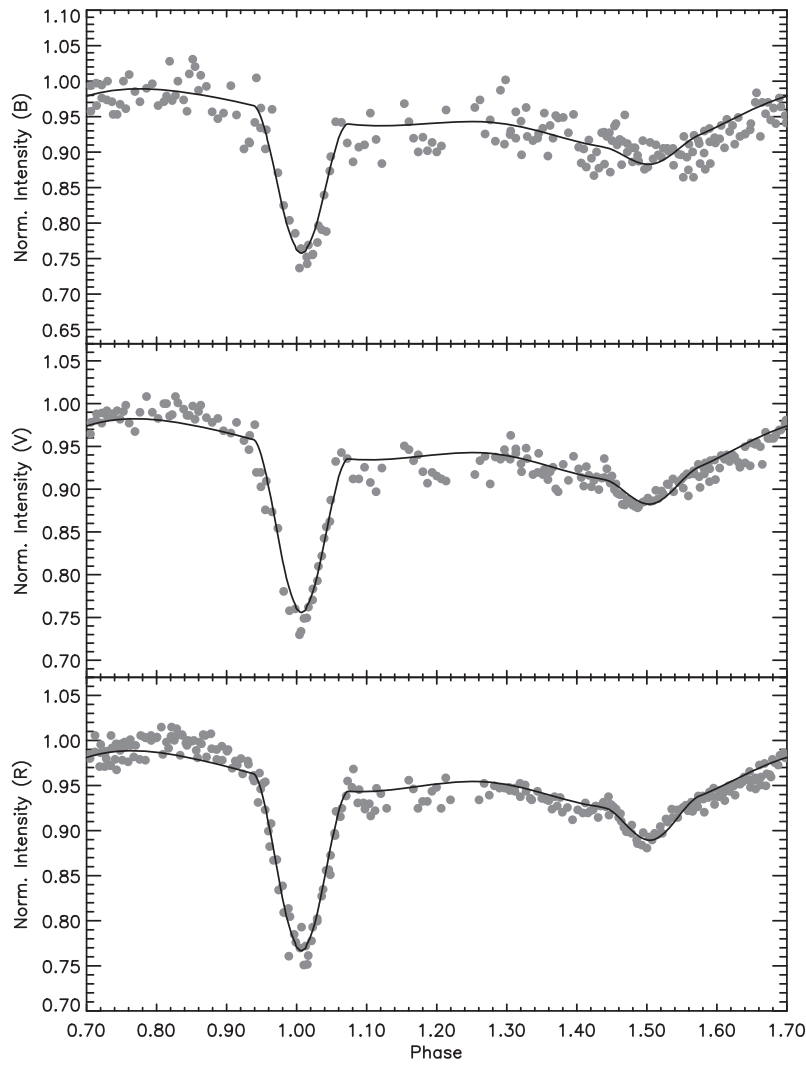

Figure 5 BVR-band light curves (filled circles) observed in 2008 and the synthetic curves (lines) derived from the light-curve solution.

\subsection{The Out-of-Eclipse Variation}

In order to explain the out-of-eclipse variation, we investigated the pre-whitened light curves in the $R$ band. Using the physical parameters, we derived a synthetic light curve of the system for an unspotted case in the $R$ band. Then, a synthetic light curve of the $R$ band was extracted from both $R$ light curves obtained in 2007 and 2008. In the second step, the $R$-band light curves, which were obtained in 2002, 2003, 2004 and 2005, presented by 
Table 3. Parameters obtained from the light-curve analysis

\begin{tabular}{ll}
\hline Parameter & Value \\
\hline$q$ & 0.55 (Fixed) \\
$i($ deg $)$ & $71.12 \pm 0.04$ \\
$T_{1}(\mathrm{~K})$ & 4040 \\
$T_{2}(\mathrm{~K})$ & $3123 \pm 16$ \\
$\Omega_{1}$ & $4.542 \pm 0.003$ \\
$\Omega_{2}$ & $3.424 \pm 0.001$ \\
$\mathrm{~L}_{1} / \mathrm{L}_{\mathrm{T}}(B)$ & $0.848 \pm 0.004$ \\
$\mathrm{~L}_{1} / \mathrm{L}_{\mathrm{T}}(V)$ & $0.909 \pm 0.002$ \\
$\mathrm{~L}_{1} / \mathrm{L}_{\mathrm{T}}(R)$ & $0.883 \pm 0.003$ \\
$g_{1}$ & 0.32 (Fixed) \\
$g_{2}$ & 0.32 (Fixed) \\
$A_{1}$ & 0.50 (Fixed) \\
$A_{2}$ & 0.50 (Fixed) \\
$x_{1, \text { bol }}$ & 0.563 (Fixed) \\
$x_{1, \mathrm{~B}}$ & 0.826 (Fixed) \\
$x_{1, \mathrm{~V}}$ & 0.799 (Fixed) \\
$x_{1, \mathrm{R}}$ & 0.747 (Fixed) \\
$x_{2, \mathrm{bol}}$ & 0.468 (Fixed) \\
$x_{2, \mathrm{~B}}$ & 0.868 (Fixed) \\
$x_{2, \mathrm{~V}}$ & 0.839 (Fixed) \\
$x_{2, \mathrm{R}}$ & 0.748 (Fixed) \\
$\left\langle r_{1}\right\rangle$ & $0.254 \pm 0.005$ \\
$\left\langle r_{2}\right\rangle$ & $0.258 \pm 0.004$ \\
Co - Lat $_{\text {Spot I }}\left({ }^{\circ}\right)$ & 110 (Fixed) \\
Long $_{\text {Spot I I }}\left({ }^{\circ}\right)$ & 188 (Fixed) \\
$R_{\text {Spot I I }}\left({ }^{\circ}\right)$ & 26 (Fixed) \\
$T_{\text {eff,Spot I }}$ & 0.95 (Fixed) \\
Co - Lat $_{\text {Spot II }}\left({ }^{\circ}\right)$ & 50 (Fixed) \\
Long $_{\text {Spot II }}\left({ }^{\circ}\right)$ & 290 (Fixed) \\
$R_{\text {Spot II }}\left({ }^{\circ}\right)$ & 15 (Fixed) \\
$T_{\text {eff,Spot II }}$ & 0.90 (Fixed) \\
\hline &
\end{tabular}

(a) Phase $=0.25$
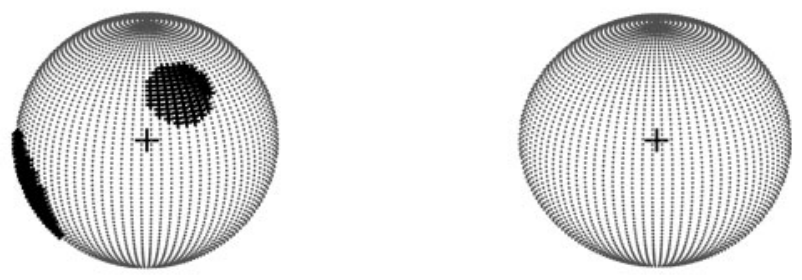

(b) Phase $=0.40$
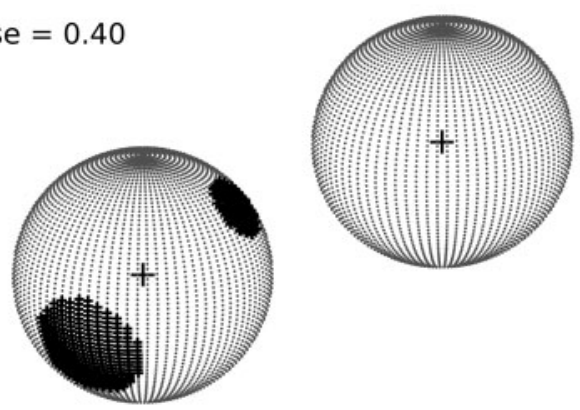

Figure 6 The geometric configurations at (a) phase 0.25 and (b) 0.40 , illustrated for DK CVn.

Terrell et al. (2005), were scanned, and the observational data were obtained from these light curves in order to compare our data with the data existing in the literature. Then, the synthetic light curve derived for unspotted case was extracted from Terrell's light curves.

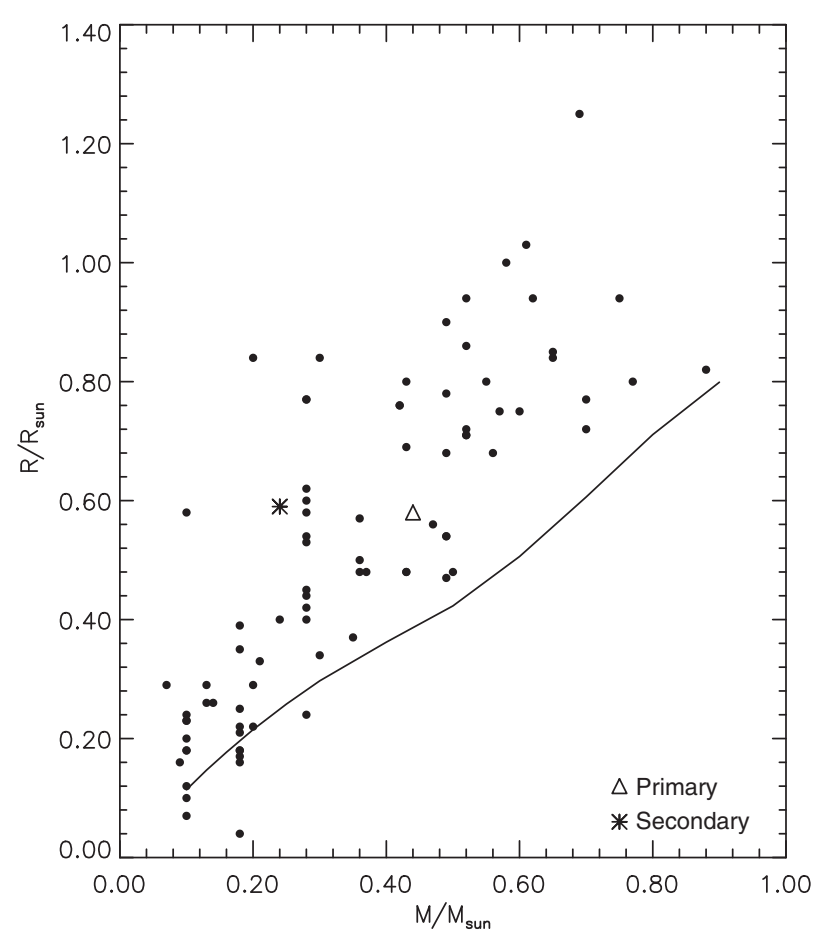

Figure 7 The location of the components of DK CVn among UV Ceti type stars in the mass-radius distribution. The filled circles represent the active stars listed in the catalogue of Gershberg et al. (1999). The asterisk represents the secondary component, while the open triangle represents the primary component of DK CVn. The line represents the ZAMS theoretical model developed by Siess, Dufour \& Forestini (2000).

The pre-whitened light curves of the season 2002, 2003, 2004, 2005, 2007 and 2008 are shown in Panels a, $\mathrm{b}, \mathrm{c}, \mathrm{d}$, e and $\mathrm{f}$ in Figure 8, respectively. If the prewhitened light curves are carefully examined, three points will be seen in the general nature of the light curves. One of them is a sinusoidal-like variation. The sinusoidal-like variations are seen in all the pre-whitened light curves as a dominant feature. The second one is that the sinusoidallike variations have an asymmetric shape. Moreover, the shapes of the sinusoidal-like variations (the minimum and maximum phases, their amplitudes and etc.) are varying from a year to the next one. Finally, there are some sudden and short-duration flares in the curves.

In the pre-whitened light curves, one asymmetric minimum is seen in the sinusoidal-like variations, generally. However, there are two minima in the pre-whitened light curve of the season 2003. Examining these sinusoidallike variations, some parameters were computed. These parameters are listed in Table 4. In the table, we listed the observing seasons, $\theta_{\min }$, the amplitude of the pre-whitened $R$ band light curves and references, respectively. The variation of the amplitude of the pre-whitened $R$ band light curves is shown in Figure 9. As seen from the figure, although the amplitude of the pre-whitened light curves are decreasing from 2002 to 2008 in the general view, a cyclic behaviour in sinusoidal form is also seen in the amplitude variation. Figure 10 is a plot of the phase of light minimum against the observing year. Apart from the 


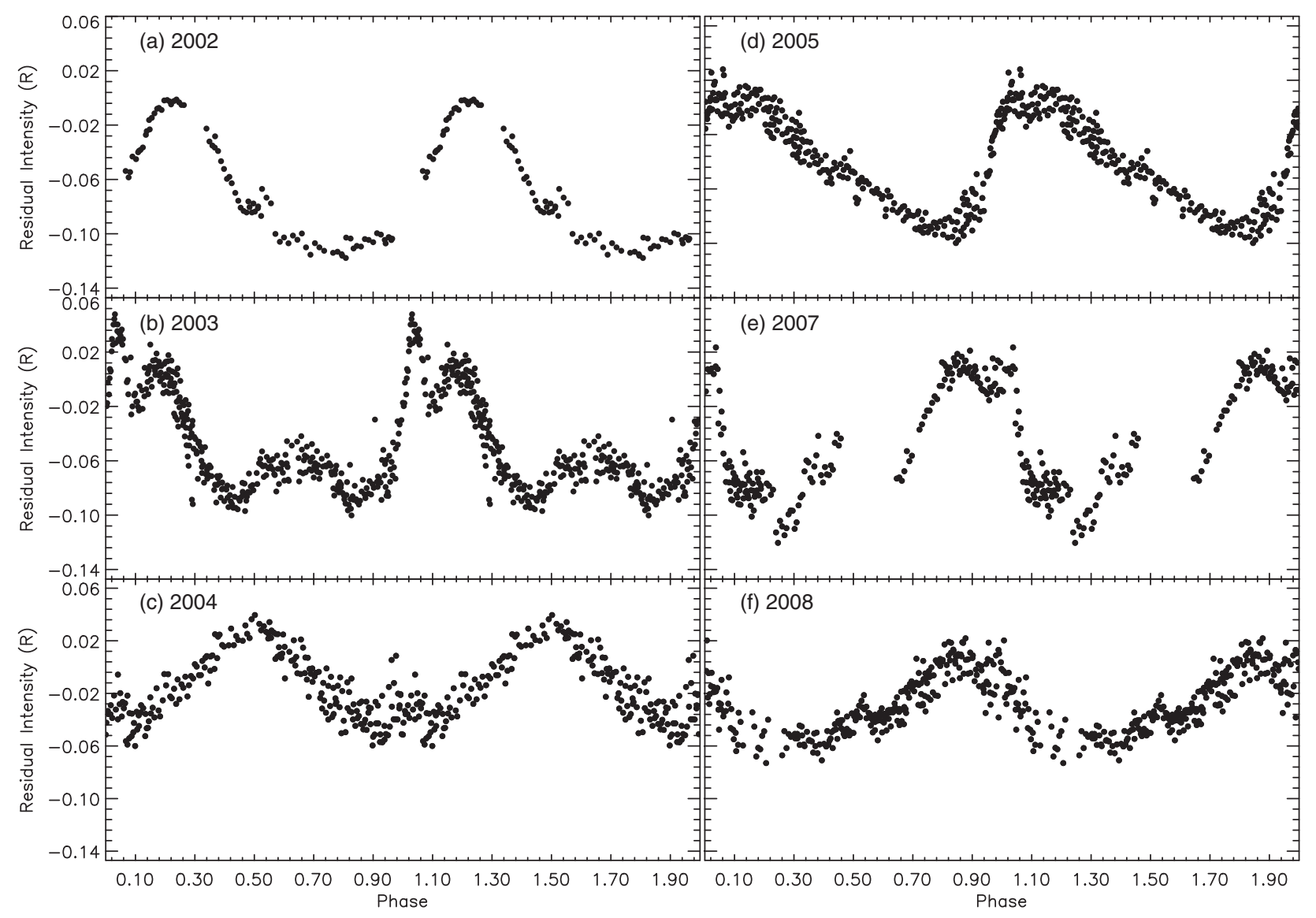

Figure 8 All the pre-whitened light curves in $R$ band (all the light curves are shown as double cycle for better visibility of light variations).

Table 4. Minimum phases and amplitudes of the sinusoidallike variation in the $\boldsymbol{R}$-band, pre-whitened light curves

\begin{tabular}{cccc}
\hline Year & $\theta_{\text {min }}$ & Amplitude in $R(\mathrm{mag})$ & Ref. \\
\hline 2002 & 0.80 & 0.139 & 1 \\
2003 & 0.90 & 0.125 & 1 \\
2004 & 1.00 & 0.079 & 1 \\
2005 & 0.80 & 0.099 & 1 \\
2007 & 0.20 & 0.115 & 2 \\
2008 & 0.18 & 0.062 & 2 \\
\hline
\end{tabular}

${ }^{1}$ Terrell et al. (2005)

${ }^{2}$ This study.

amplitude, the minima phases $\left(\theta_{\min }\right)$ also exhibit a variation. The minima phases are separating in two mean longitudes. The $\theta_{\text {min }}$ determined from such light curves would give the effective longitude of the spot or spots group. There is an indication of two effective longitudes in which the spots are generally formed. One of them is around 0 P.80, while the other is around 0.20 .

\subsection{Flare Activity}

Apart from both eclipses and the sinusoidal-like variations out of eclipse, DK CVn also exhibits the flare activity. In this study, we detected one flare in the observations of 2007 and two flares in 2008. The flare shown in Figure 11 was detected in 2007 and exhibits itself in each band, while the flares detected in 2008 exhibits themselves in just $B$ and $V$ bands. They are shown in Figure 12. These two flares can not be detected in $R$ band due to their lower powers. In addition to the flares detected in this study, the flare detected in $B$ band by Terrell et al. (2005), was scanned and we got its observational data as well. Terrell's flare is shown in Figure 13. The flare parameters were calculated for each flare, and we list them in Table 5. In the table, the observing season, observing band, HJD of the flare maxima, flare rise time (s), flare decay time (s), flare total duration (s), flare equivalent duration (s), flare amplitude (mag) are listed in each column, respectively. In the last column, we noted which study the flare data are belonged.

To calculate the flare parameters, we used the method described by Dal \& Evren (2010). However, a different way was followed to determine the quiescent level of the brightness due to the eclipsing binary nature of DK CVn. Using the synthetic light curve; we obtained the quiescent level of the brightness for each phase. In order to test whether the method is correctly working or not, we compared the light curves observed in the consecutiveclose nights and the synthetic light curve. Some examples for these comparisons are shown in Figures 11 and 12. As seen from the figures, DK CVn was observed two or more times in each phase intervals in close dates. Sometimes a 


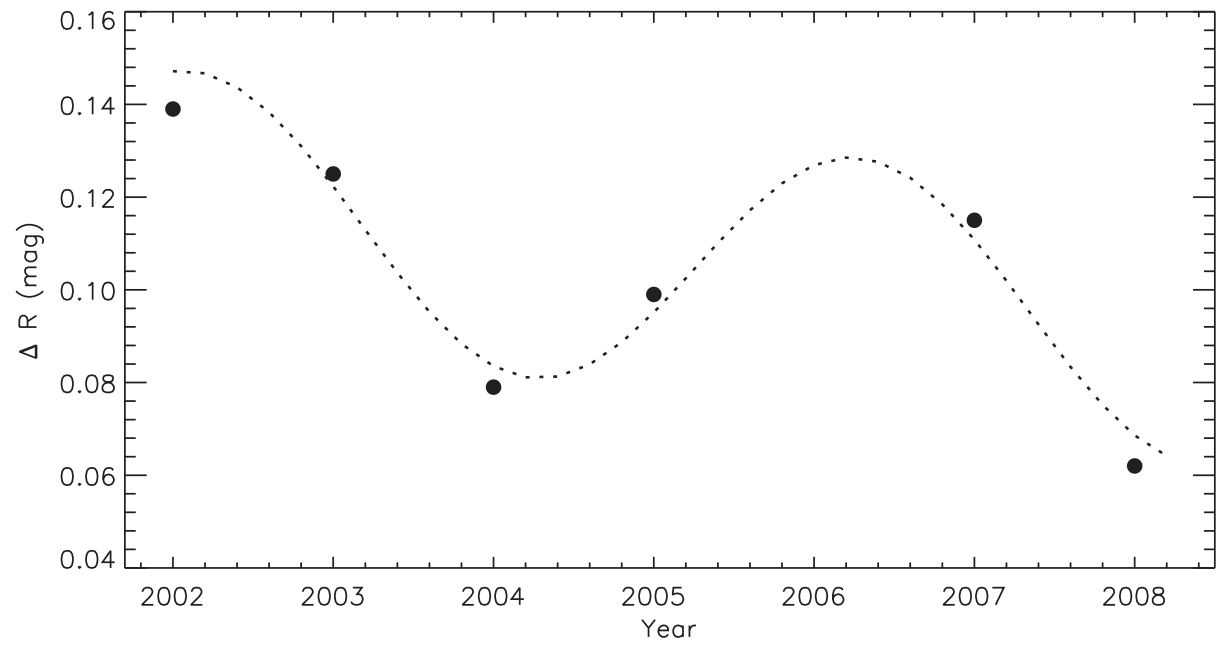

Figure 9 The variation of the amplitude of the variation at out of eclipse, throughout the years. The dashed line represents the fits of the variations in the figure.

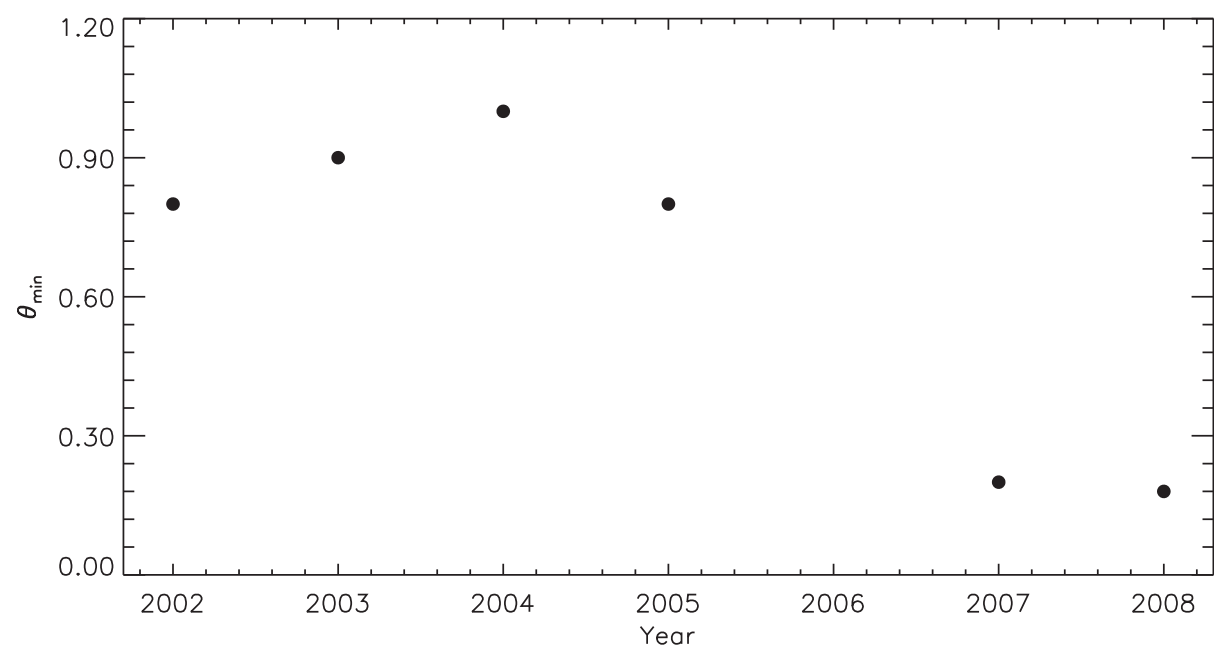

Figure 10 The minimum phases for out-of-eclipse variation throughout the years.

flare was detected in one observing night, while no flare was detected in the same phase interval in another-close observing night. Comparing these observations with the synthetic light curve aids to determine the actual flare light variation.

As seen from Figure 11, a flare was detected around the primary minima on 2007 April 18. The system was observed in the same phase interval on 2007 March 8, but no flare was detected. The flare detected on 2007 April 18, distorted almost the shape of the primary minimum. The similar case is seen in the observations of 2008. Two flares were detected on 2008 March 16, while no flare was detected on 2008 March 2. The flare taken from Terrell et al. (2005), is shown in Figure 13. We compared Terrell's flare light curve with only the synthetic light curve due to absence of another observation in a consecutive-close night.

Using Equations 2 and 3 described in the method developed by Gershberg (1972), the flare equivalent durations and flare energies can be calculated.
In Equation 2, $I_{0}$ is the intensity of the star at quiescence and $I_{\text {flare }}$ is the intensity during flare:

$$
P=\int \frac{\left(I_{\text {flare }}-I_{0}\right)}{I_{0}} \mathrm{~d} t
$$

and

$$
E=P \times L,
$$

where $E$ is the flare energy, $P$ is the equivalent duration of the flare given by Equation 2, and $L$ is the luminosity of the stars at quiescence.

To understand whether the flares observed from DK $\mathrm{CVn}$, which is an eclipsing binary system, are similar to the flares occurring on the surface of UV Ceti type stars, DK CVn's flares were compared with $B$-band flares of five UV Ceti type stars presented by Dal \& Evren (2011). To be able to compare them, first of all, following the method developed by Dal \& Evren (2011), the 

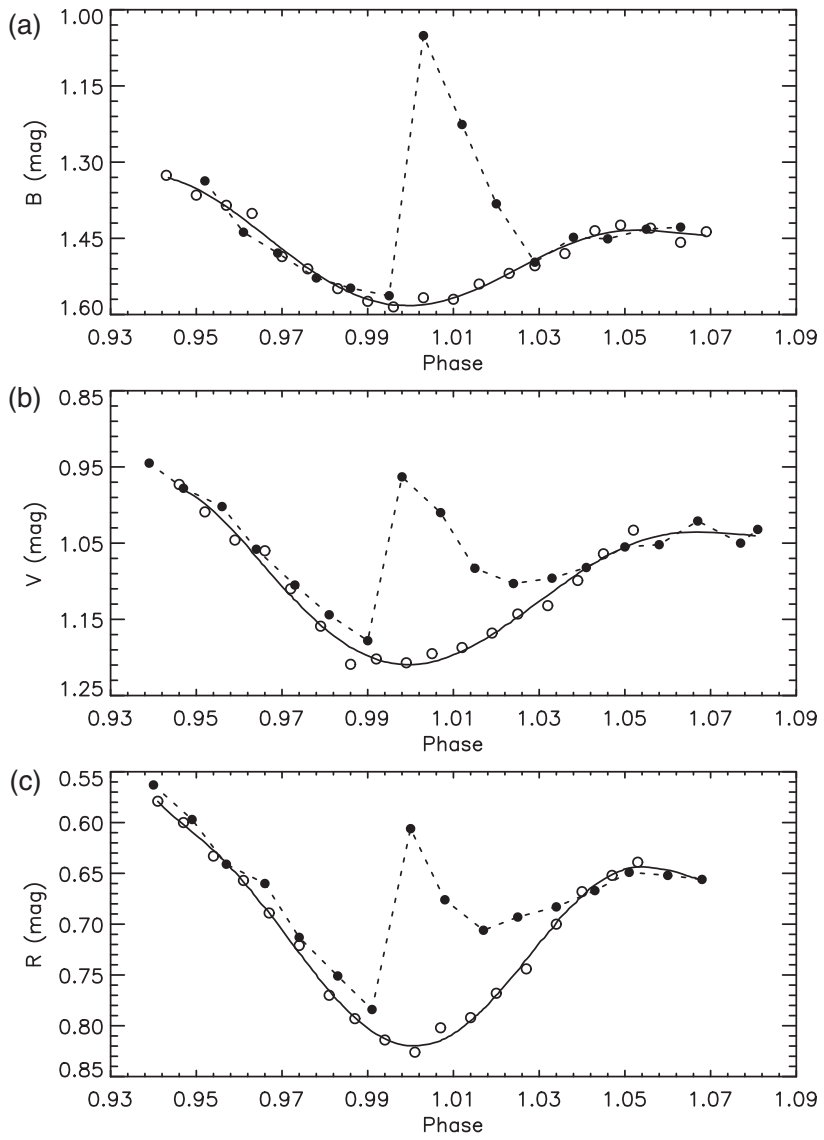

Figure 11 The fast-flare sample detected around primary minimum on 2007 April 18. Open circles represent the observations on 2007 April 18, while filled circles represent the observations on 2007 March 8. The line represents the synthetic light curve.

distribution of the flare equivalent durations versus flare total durations were derived for the $B$-band flares of DK CVn. Using spss (v17.0) software (Green et al. 1999) and GRAHPPAD PRISM (v5.02) software (Motulsky 2007; Dawson \& Trapp 2004), the best model function was determined. Using the least-squares method, regression calculations showed that the best model function of distribution is the OPEA function (Motulsky 2007; Spanier \& Oldham 1987) given by:

$$
y=y_{0}+\left(\text { Plateau }-y_{0}\right) \times\left(1-e^{-k x}\right)
$$

The derived OPEA model of DK CVn's flares is shown in Figure 14. Using this model, some parameters of the flare equivalent duration, such as $y_{0}$, Plateau, $K$, Span, Half - Life values, were computed. In the OPEA model function given by Equation 4, the $y$ values were taken as flare equivalent duration in logarithmic scale, while $x$ values were taken as flare total durations. The parameter $y_{0}$ is the lowest flare equivalent duration obtained in logarithmic scale, while the parameter Plateau is the upper limit the flare equivalent durations can reach.

According to Equation 3, Plateau value depends only on flare energy, while $y_{0}$ value depends on the brightness of the target and sensitivity of the optical system, as well
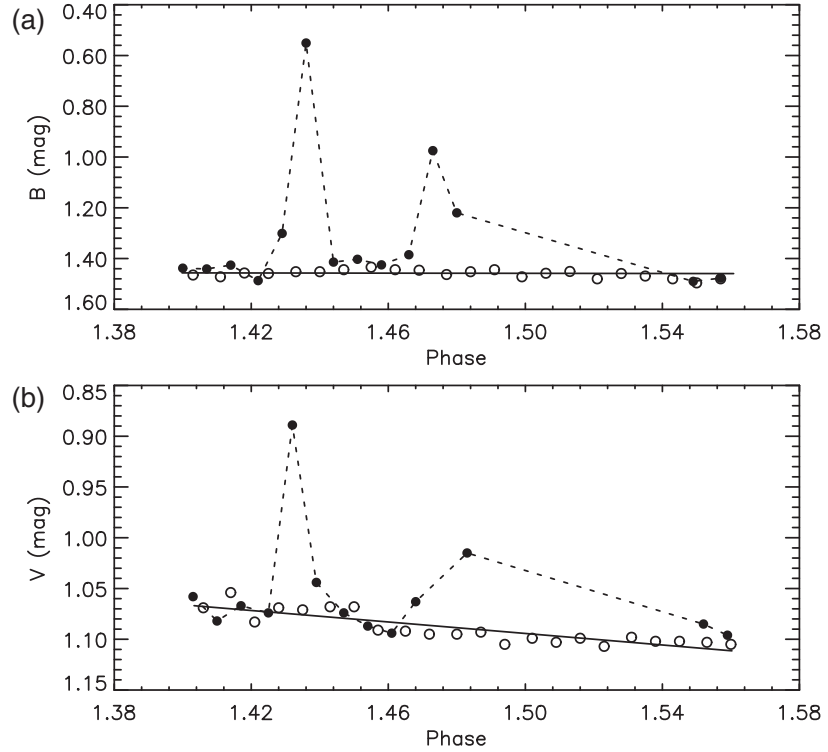

Figure 12 Two fast-flare samples detected on 2008 March 16 . Open circles represent the observations on 2008 March 16, while filled circles represent the observations on 2008 March 2. The line represents the synthetic light curve.

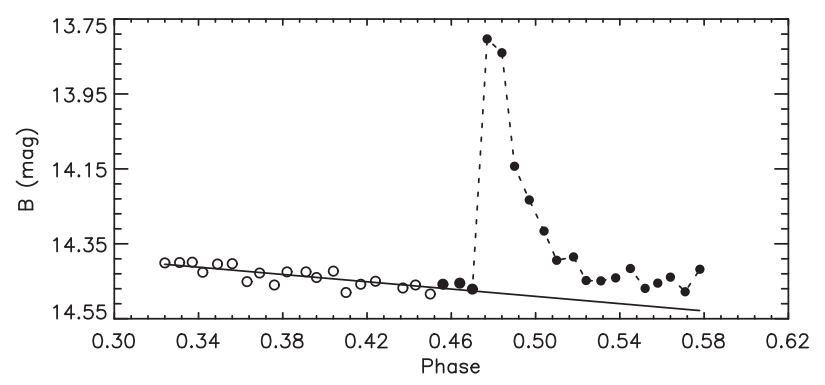

Figure 13 The fast-flare sample detected by Terrell et al. (2005) in $B$ band.

as flare power. The parameter $K$ is a constant value depending on $x$ values. The Span value is a difference between the Plateau and $y_{0}$ values. The Half - Life value is half of the first $x$ values, where the model reaches the Plateau value. In other words, it is half of the flare total duration, where flares with the highest energy start to be seen. The Half - Life value is an indicator of the duration the flare process occurring on the surface of a star needs to reach the saturation. As seen from the distribution of flare equivalent durations, the flare equivalent durations increase with the flare total duration until a specific total duration value, and then the flare equivalent durations became constant, no matter how long the flare total duration is. The Half - Life value is half of this specific total duration value. All the parameters computed from the OPEA model are listed in Table 6. In order to test whether the Plateau value is statistically acceptable for this distribution of the flare equivalent durations, using the Independent Sample t-Test (hereafter t-Test, Motulsky 2007; Dawson \& Trapp 2004; Green et al. 1999), we compute the mean average value of the flares, which are located in the Plateau phase of the model. The 
Table 5. Flare parameters from this study and from Terrell et al. (2005)

\begin{tabular}{|c|c|c|c|c|c|c|c|c|}
\hline Year & Filter & $\begin{array}{l}\text { HJD of maxima } \\
(+2400000)\end{array}$ & $\begin{array}{l}\text { Rise } \\
\text { time (s) }\end{array}$ & $\begin{array}{l}\text { Decay } \\
\text { time (s) }\end{array}$ & $\begin{array}{c}\text { Total } \\
\text { duration (s) }\end{array}$ & $\begin{array}{l}\text { Equivalent } \\
\text { duration (s) }\end{array}$ & $\begin{array}{l}\text { Amplitude } \\
\text { (mag) }\end{array}$ & Ref. \\
\hline 2005 & $B$ & 53394.90620 & 568 & 4315 & 4884 & 929.1551 & 0.668 & 1 \\
\hline 2007 & $B$ & 54209.48337 & 363 & 1476 & 1839 & 429.6051 & 0.516 & 2 \\
\hline 2008 & $B$ & 54542.31363 & 626 & 940 & 1566 & 595.2623 & 0.901 & 2 \\
\hline 2008 & $B$ & 54542.33176 & 313 & 626 & 939 & 264.5768 & 0.488 & 2 \\
\hline 2007 & $V$ & 54209.48084 & 363 & 1476 & 1839 & 214.9586 & 0.244 & 2 \\
\hline 2008 & $V$ & 54542.31137 & 313 & 627 & 940 & 68.9973 & 0.180 & 2 \\
\hline 2007 & $R$ & 54209.48168 & 363 & 1476 & 1839 & 189.9065 & 0.220 & 2 \\
\hline
\end{tabular}

${ }^{1}$ Terrell et al. (2005)

${ }^{2}$ This study.

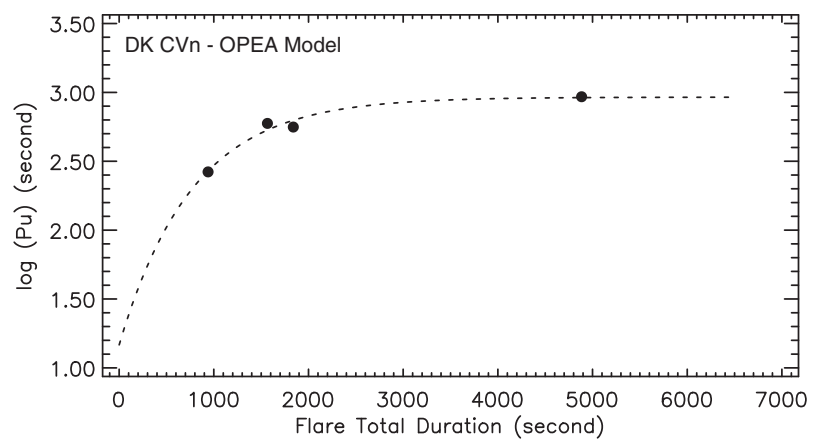

Figure 14 The distribution of the flare equivalent durations versus the flare total durations for DK CVn flares (filled circles). The OPEA model (dashed line) derived for this distribution.

Table 6. Parameters computed from the OPEA model of the flares in DK CVn

\begin{tabular}{ll}
\hline Parameter & Value \\
\hline Max. Rise Time (s) & 626 \\
Max. Tot. Time (s) & 4884 \\
Plateau (s) & $2.965 \pm 0.07374$ \\
$y_{0}(\mathrm{~s})$ & $1.167 \pm 0.1555$ \\
K(s) & $0.001289 \pm 0.000495$ \\
Span (s) & $1.798 \pm 0.1243$ \\
Half - Life (s) & 537.7 \\
Mean (s) & 2.731 \\
\hline
\end{tabular}

resultant mean average value is listed in the last row of Table 6.

All the parameters listed in Table 6 were compared with the parameters given by Dal \& Evren (2011) for five UV Ceti type stars. In the comparison, we assume that the flares are occurring on the surface of the cool component of the system. The light curve analysis of the system indicates that the temperature of the cool component is $3123 \mathrm{~K}$. According to Tokunaga (2000), this temperature is corresponding to $B-V=1.630 \mathrm{mag}$ as the colour index. All the comparisons are shown in Figures 15 and 16.

As seen from Figures Figures 15 and 16, in fact, the flares detected in the observations of DK CVn seem to have a same nature with the flares detected from UV Ceti type stars. Moreover, our assumption also seems to be correct, because the parameters are in agreement with its analogue according to assumed temperature.

\section{Results and Discussion}

In this study, we obtained the light curves of an eclipsing binary system, DK CVn, in two observing seasons, and we analysed the $B V R$ light curves obtained in 2008 to find the physical properties of DK CVn. In addition, using Kepler's third law under some assumptions, we derived absolute parameters for the system. The observations demonstrate that the radii are generally larger than the expected values. We compared the radii of DK CVn's components with the known active stars and a model. The radii of the active stars, which exhibit spot or flare activity or both of them, are dramatically larger than the values given by the model developed for the stars with $Z=0.02$ by Siess, Dufour \& Forestini (2000).

The components of DK CVn are also in agreement with other active stars, listed in the catalogue of Gershberg et al. (1999). According to several theoretical models and observational studies (Ribas 2006; Chabrier, Galla \& Baraffe 2007; Morales, Ribas \& Jordi 2008; Morales et al. 2010), the case seen in Figure 7 is a well known phenomenon for low-mass active stars. For instance, YY Gem (Torres \& Ribas 2002), CU Cam (Ribas 2003) and CM Dra (Morales et al. 2009) are the most popular system for this case. There are several similarities between these three systems and DK CVn. Firstly, all of them exhibit the spot and flare activities, and they consist of the low mass components.

The observations in $B$ and $V$ bands demonstrate that the system exhibits flare activity. Considering the effective temperature, we assumed that the flares occur on the secondary component. The derived parameters demonstrate that DK CVn's flares seem to behave in the same way with the flares of UV Ceti stars. The maximum energy level of the flares seen in the DK CVn system is in agreement with the analogues of UV Ceti stars from the late spectral types. Consequently, the flares detected from DK CVn must be produced by the same process occurring on the surface of an UV Ceti star.

Considering the flare activity exhibited by DK CVn system, both components could be chromospherically active stars. In addition, the spectral types of both 

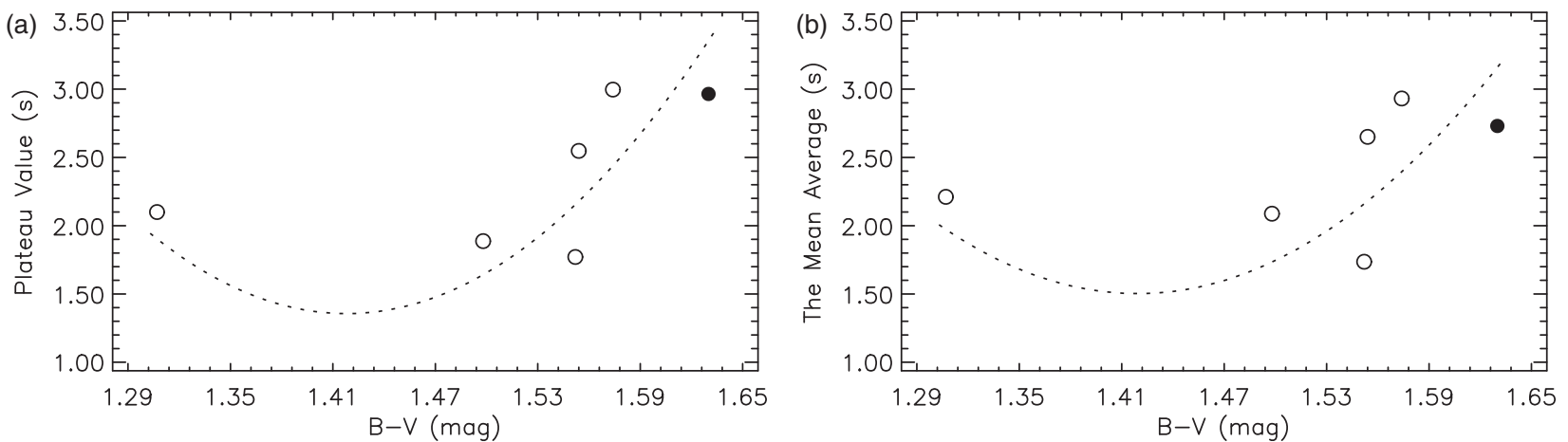

Figure 15 The variations of (a) the Plateau values derived from the OPEA models and (b) the mean equivalent durations computed by t-Test analyses versus $B-V$ colour index. In the figures, the filled circles represent the DK CVn, while open circles represent five UV Ceti type stars taken from Dal \& Evren (2011). The dashed lines are just used to show the variation trend.
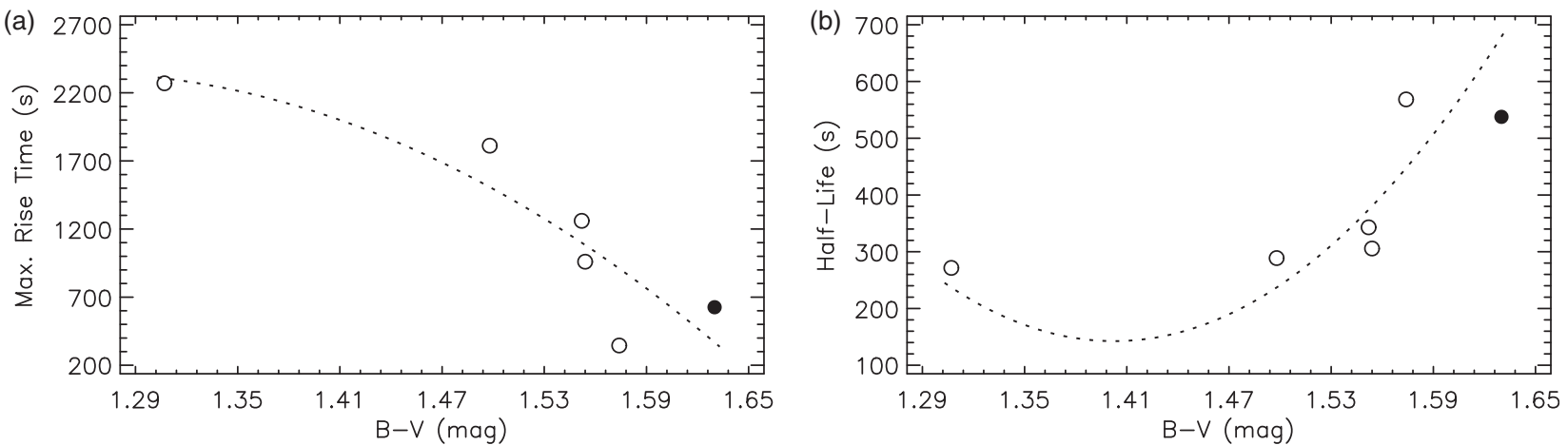

Figure 16 The variations of (a) the maximum flare rise times and (b) the Half - Life values versus $B-V$ colour index. In the figures, all the symbols are the same with Figure 15.

components are consistent with chromospheric activity. Another support comes from the light curve analysis. The observed light curve can be modelled with two cool spots on the one of the components due to the asymmetry seen in the shape of the curve. We assumed that the spots are located on the primary component, and we analysed the light curves with this assumption. According to its effective temperature, the secondary component would be very close to the border of the fully convective area of an M dwarfs. Although fully convective $\mathrm{M}$ dwarfs exhibit very strong flare activity, a few of them just exhibit spot activity. However, K dwarfs are generally stars which can exhibit spot activity. This is why we assumed the spotted star is the primary component.

Considering the sum of weighted squared residuals, we found from the $B V R$ light curve analyses that there are a large spot and a small one on the primary component. The larger spot with a temperature factor of 0.95 is located in longitude of $188^{\circ}$, while the small one with a temperature factor of 0.90 is located in longitude of $290^{\circ}$. It is well known that the longitudes of the spotted areas can be found exactly from the light curves obtained with photometric observations. However, the co-latitude, radius and temperature factor of the spot are not very well defined. A similar synthetic curves fitting the observations can be derived with some different values of these parameters.
In this study, when we considered the sum of weighted squared residuals, the radii, co-latitudes and temperature factors derived for the spots give one of the best synthetic curves, which are seen in Figure 5. They are statistically acceptable, and the physical parameters of the components are also acceptable in the astrophysical sense with these spot parameters.

However, the asymmetry and variation of the prewhitened light curves reveal that the cool spots vary with time, as well. Although the spots can sometimes change over the course of a few months (as it is seen from NSVS data) generally the spots do not seem to change on short time-scales. The spots occur at two longitudes, i.e. in phases $0.80-0.90$ and $0.00-0.20$, which suggests that the spots are concentrated at two active longitudes. The amplitude of the pre-whitened light curves demonstrated this variation very well. In general, the amplitude of the out-of-eclipse variations has been decreasing since 2002, while a cyclic variation in sinusoidal shape is also seen, combining with general decreasing.

The phases of the minima change dramatically in this system. The variations of the pre-whitened light curves indicate that the spotted areas are not stable on the component. In the pre-whitened light curves of the season 2003, two minima are seen separately from each other. In the season 2005, the pre-whitened light curve has a very 
strong asymmetry. The variations seen out of eclipses are similar to the variations exhibiting by the young-fastrotating stars, such as YY Gem, ER Vul, SV Cam, CU Cam and CM Dra (Strassmeier 2009; Torres \& Ribas 2002; Ribas 2003; Morales et al. 2009). Therefore, the spotted component (and the system) could be a young star. On the other hand, it must be noted that there are many systems that exhibit unexpected behaviour when subjected to this approach (Rocha-Pinto, Castilho \& Maciel 2002).

In brief, the variation seen at out of eclipse could be due to the cool spots gathering into two separate longitudes on the surface of the primary component. Thus, this variation is caused by the rotational modulation due to chromospheric activity. Consequently, DK CVn seems to be an analogue of RS CVn type stars. In addition, both spot and flare activities indicate that the system has high level chromospheric activity.

\section{Acknowledgments}

The authors acknowledge generous allotments of observing time at the Ege University Observatory. We thank Dr. Dirk Terrell for reviewing the manuscript and his useful comments that have contributed to the improvement of the paper.

\section{References}

Akerlof, C. et al., 2000, AJ, 119, 1901

Brát, L., Zejda, M. \& Svoboda, P., 2007, OEJV, 74, 1

Brát, L. et al., 2008, OEJV, 94, 1

Brát, L. et al., 2009, OEJV, 107, 1

Chabrier, G., Gallardo, J. \& Baraffe, I., 2007, A\&A, 472, L17

Dal, H. A. \& Evren, S., 2010, AJ, 140, 483

Dal, H. A. \& Evren, S., 2012, NewA, 17, 399

Dawson, B. \& Trapp, R. G., 2004, Basic and Clinical Biostatistics (USA: The McGraw-Hill Companies Inc. Press)

Diethelm, R., 2001, IBVS, 5060, 1
Gershberg, R. E., 1972, ApSS, 19, 75

Gershberg, R. E., Katsova, M. M., Lovkaya, M. N., Terebizh, A. V. \& Shakhovskaya, N. I., 1999, A\&AS, 139, 555

Green, S. B., Salkind, N. J. \& Akey, T. M., 1999, Using SPSS for Windows: Analyzing and Understanding Data (London: Prentice Hall Press), 50

Hardie, R. H., 1962, in Astronomical Techniques, ed. W. A. Hiltner (Chicago: University of Chicago Press), 178

Kazarovets, E. V., Kireeva, N. N., Samus, N. N. \& Durlevich, O. V., 2003, IBVS, 5422, 1

Landolt, A. U., 1983, AJ, 88, 439

Landolt, A. U., 1992, AJ, 104, 340

Lucy, L. B., 1967, ZA, 65, 89

Malkov, O. Yu., Oblak, E., Snegireva, E. A. \& Torra, J., 2006, A\&A 446,785

Morales, J. C., Ribas, I. \& Jordi, C., 2008, A\&A, 478, 507

Morales, J. C. et al., 2009, ApJ, 691, 1400

Morales, J. C., Gallardo, J., Ribas, I., Jordi, C., Baraffe, I. \& Chabrier, G., 2010, ApJ, 718, 502

Motulsky, H., 2007, GraphPad Prism 5: Statistics Guide (San Diego: GraphPad Software Inc. Press)

Prša, A. \& Zwitter, T., 2005, ApJ, 628, 426

Ribas, I., 2003, A\&A, 398, 239

Ribas, I., 2006, ApSS, 304, 89

Rocha-Pinto, H. J., Castilho, B. V. \& Maciel, W. J., 2002, A\&A, 384,912

Rucinski, S. M., 1969, AcA, 19, 245

Siess, L., Dufour, E. \& Forestini, M., 2000, A\&A, 358, 593

Sipahi, E., Dal, H. A. \& Özdarcan, O, 2009, IBVS, 5904, 1

Spanier, J. \& Oldham, K. B., 1987, An Atlas of Function (Washington, DC: Hemisphere Publishing Corporation Press), 233.

Strassmeier, K. G., 2009, A\&ARv, 17, 251

Terrell, D. et al., 2005, IBVS, 5642, 1

Tokunaga, A. T., 2000, in Allen's Astrophysical Quantities, ed. A. N. Cox (4th ed.; New York: Springer), 143

Torres, G. \& Ribas, I., 2002, ApJ, 567, 1140

van Hamme, W., 1993, AJ, 106, 2096

Wilson, R. E., 1990, ApJ, 356, 613

Wilson, R. E. \& Devinney, E. J., 1971, ApJ, 166, 605

Woźniak, P. R. et al., 2004, AJ, 127, 2436

Zacharias, N., Monet, D. G., Levine, S. E., Urban, S. E., Gaume, R. \& Wycoff, G. L., 2004, AAS, 205, 4815 